\title{
A TRANSMISSION PROBLEM FOR THERMOELASTIC PLATES
}

\author{
BY
}

JAIME E. MUÑOZ RIVERA (National Laboratory for Scientific Computation, Department of Applied Mathematic, Av. Getúlio Vargas 333, Quitandinha, 25651-070, Petrópolis, RJ, Brazil, and IM, Federal University of Rio de Janeiro)

AND

HIGIDIO PORTILLO OQUENDO (Federal University of Paraná, Department of Mathematics, Politechnic Center, P. O. Box 19081, Jardim das Americas, CEP 81531-990 Curitiva, Paraná, Brazil)

Abstract. In this paper we study a transmission problem for thermoelastic plates. We prove that the problem is well-posed in the sense that there exists only one solution which is as regular as the initial data. Moreover, we prove that the local thermal effect is strong enough to produce uniform rate of decay of the solution. More precisely, there exist positive constants $C$ and $\gamma$ such that the total energy $E(t)$ satisfies

$$
E(t) \leq C E(0) e^{-\gamma t} \text {. }
$$

1. Introduction. From the point of view of applications, the suppression of vibration of elastic structures is one of the important topics in material science. For example, engineers at the Ford Motor Company designed a constrained-layer damping patch which was attached to an elastic plate. They compared the natural frequencies and mode shape of the plate with and without the patch to ascertain the effect of the patch. Due to the presence of the patches, the material properties of the structure, such as the elasticity moduli, damping coefficient, and Poisson ratio, are changed (see [14]). In particular, jump discontinuity at the location of the edges of the patches is usually introduced to these properties. In this direction we will consider the model which defines the oscillation of a plate which is composed of a thermoelastic part and an elastic part. This means that the thermal constant is discontinuous on the plate, positive over the thermoelastic part, and vanishing on the elastic part.

We will consider that the plate, in equilibrium, occupies a region $\Omega$ which is a bounded open set in $\mathbb{R}^{n}$ with boundary $\partial \Omega=\Gamma_{1} \cup \Gamma_{2}$ where $\Gamma_{1}, \Gamma_{2}$ are two smooth surfaces such that $\bar{\Gamma}_{1} \cap \bar{\Gamma}_{2}=\emptyset$. We assume that the plate's particles in $\Omega_{1}$ are sensitive to change of temperature and in its complementary part, $\Omega_{2}=\Omega \backslash \Omega_{1}$, they are not. Let us denote

Received March 19, 2002.

2000 Mathematics Subject Classification. Primary 74F05, 74K20, 35B40.

Supported by a grant of CNPq (Brazil). 
by $\Gamma_{0}$ the common smooth surface between $\Omega_{1}$ and $\Omega_{2}$; a region $\Omega$ of this type is given by Fig. 1.

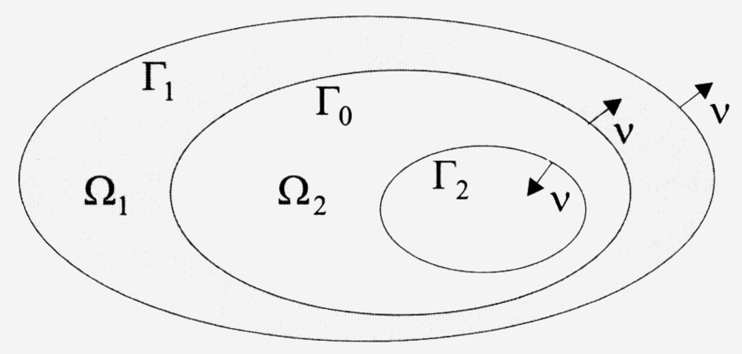

FIG. 1. The set $\Omega$

Denoting by $u(x, t)$ and $v(x, t)$ the vertical displacements of the plate and by $\theta(x, t)$ the difference of temperature, the corresponding model can be written as follows:

$$
\begin{aligned}
\rho_{1} u_{t t}-\gamma_{1} \Delta u_{t t}+\beta_{1} \Delta^{2} u+\mu \Delta \theta & =0 \text { in } \Omega_{1} \times \mathbb{R}^{+}, \\
\rho_{0} \theta_{t}-\beta_{0} \Delta \theta+\gamma_{0} \theta-\mu \Delta u_{t} & =0 \text { in } \Omega_{1} \times \mathbb{R}^{+}, \\
\rho_{2} v_{t t}-\gamma_{2} \Delta v_{t t}+\beta_{2} \Delta^{2} v & =0 \text { in } \Omega_{2} \times \mathbb{R}^{+} .
\end{aligned}
$$

We assume that the plate is clamped on the surfaces $\Gamma_{1}, \Gamma_{2}$, i.e.,

$$
u=\frac{\partial u}{\partial \nu}=0 \quad \text { on } \quad \Gamma_{1} \times \mathbb{R}^{+}, \quad v=\frac{\partial v}{\partial \nu}=0 \quad \text { on } \quad \Gamma_{2} \times \mathbb{R}^{+} .
$$

The transmission condition on the interface $\Gamma_{0}$ is given by

$$
\begin{aligned}
& u=v, \quad \frac{\partial u}{\partial \nu}=\frac{\partial v}{\partial \nu}, \quad \beta_{1} \Delta u+\mu \theta=\beta_{2} \Delta v \quad \text { on } \quad \Gamma_{0} \times \mathbb{R}^{+}, \\
& -\gamma_{1} \frac{\partial u_{t t}}{\partial \nu}+\beta_{1} \frac{\partial \Delta u}{\partial \nu}+\mu \frac{\partial \theta}{\partial \nu}=-\gamma_{2} \frac{\partial v_{t t}}{\partial \nu}+\beta_{2} \frac{\partial \Delta v}{\partial \nu} \quad \text { on } \quad \Gamma_{0} \times \mathbb{R}^{+} \text {. }
\end{aligned}
$$

We consider the following condition for the temperature:

$$
\theta=0 \quad \text { on } \quad \Gamma_{0} \times \mathbb{R}^{+}, \quad \frac{\partial \theta}{\partial \nu}+\lambda \theta=0 \quad \text { on } \quad \Gamma_{1} \times \mathbb{R}^{+}
$$

and the initial data

$$
\begin{array}{lll}
u(0)=u_{0}, & u_{t}(0)=u_{1}, \quad \theta(0)=\theta_{0} & \text { in } \quad \Omega_{1}, \\
v(0)=v_{0}, & v_{t}(0)=v_{1} & \text { in } \quad \Omega_{2} .
\end{array}
$$

Here, the coefficients $\rho_{i}, \gamma_{i}, \beta_{i}$, and $\lambda$ are positive, $\mu$ is different to zero, and $u_{0}, u_{1}, \theta_{0}, v_{0}$, $v_{1}$ are prescribed functions. To fix ideas we consider $\mu$ positive.

Controllability for transmission problems were studied by several authors; for example, the transmission problem for the wave equation was studied by Lions [6]. He applied the Hilbert Uniqueness Method (HUM) to show the exact controllability. Later, Lagnese [5], also applying HUM, extended this result; he showed the exact controllability for a class of hyperbolic systems which include the transmission problem for homogeneous anisotropic materials. The exact controllability for the plate equation was proved by Liu and Williams [9] and Aassila [1]. 
Concerning asymptotic stability, second order transmission problems were studied by Rivera and Oquendo [11], Liu and Williams [8], and Rivera and Ma To Fu [10], while for beams we have the works of Rivera and Oquendo [12, 13]. Thermoelastic plates were studied by Lagnese, Avalos, and Lasiecka. In [4], Lagnese obtained the exponential decay of solutions with the aid of a further mechanical dissipation on the boundary and in [2], Avalos and Lasiecka obtained the same result removing the boundary dissipation. It seems to us that there is no result concerning the asymptotic stability of solution for plates made of different types of materials. So to fill this gap we study this topic here.

The main result of this paper is to show that the dissipation given by the thermal part of the plate is strong enough to produce uniform stability of the solution, no matter how thin it is. To attain this goal we will assume that the material type in $\Omega_{1}$ is more stiff than that in $\Omega_{2}$, that is

$$
\rho_{1} \geq \rho_{2}, \quad \gamma_{1} \geq \gamma_{2} \quad \text { and } \quad \beta_{1} \leq \beta_{2}
$$

Additionally, some geometric assumptions on $\Omega$ will be taken into account, as for example

$$
\begin{array}{lll}
\left(x-x_{0}\right) \cdot \nu(x) \geq \delta_{0} & \text { on } \quad \Gamma_{0} \\
\left(x-x_{0}\right) \cdot \nu(x) \leq 0 & \text { on } \quad \Gamma_{2}
\end{array}
$$

for some $x_{0} \in \mathbb{R}^{n}$ and $\delta_{0}>0$ small. In these conditions we will show that the total energy associated to the model decays exponentially as time goes to infinity. The idea we use to achieve our result is based on the energy method; to do so, we need that the solution enjoys the regularity property. Therefore, in the next section of the article, we will show that the solution of the above system has the m-regularity result. One of the main difficulties we have in showing the exponential decay is due to the boundary conditions. We avoid them using some localized multipliers and some technical ideas involving the compact embedding of the spaces $H^{m-1} \subset H^{m}$.

The remaining part of this article is organized as follows. In the next section we will show that the problem is well-posed in the sense of existence, uniqueness, and regularity of the solution. To do this we will use the semigroup approach. Finally in Sec. 3 we will prove that the solution of the system decays exponentially to zero.

2. Existence of solutions. To find a solution for the problem (1.1)-(1.8), we shall use the semigroup approach. Let us start analyzing the associated stationary problem. First we shall introduce some notation. Let us consider the following Hilbert spaces

$$
\begin{gathered}
H_{\mathrm{T}}^{1}:=\left\{\left(\phi_{1}, \phi_{2}\right) \in H^{1}\left(\Omega_{1}\right) \times H^{1}\left(\Omega_{2}\right): \phi_{i}=0 \text { on } \Gamma_{i}, \phi_{1}=\phi_{2} \text { on } \Gamma_{0}\right\} \\
H_{\mathrm{T}}^{2}:=\left\{\left(\phi_{1}, \phi_{2}\right) \in\left[H^{2}\left(\Omega_{1}\right) \times H^{2}\left(\Omega_{2}\right)\right] \cap H_{\mathrm{T}}^{1}: \frac{\partial \phi_{i}}{\partial \nu}=0 \text { on } \Gamma_{i}, \frac{\partial \phi_{1}}{\partial \nu}=\frac{\partial \phi_{2}}{\partial \nu} \text { on } \Gamma_{0}\right\} \\
H_{\mathrm{D}}^{1}:=\left\{\phi \in H^{1}\left(\Omega_{1}\right): \phi=0 \quad \text { on } \quad \Gamma_{0}\right\}, \quad H^{0}:=L^{2}\left(\Omega_{1}\right),
\end{gathered}
$$


with the following inner products:

$$
\begin{aligned}
\left(\left\{w_{1}, w_{2}\right\},\left\{\phi_{1}, \phi_{2}\right\}\right)_{H_{\mathrm{T}}^{1}}:= & \int_{\Omega_{1}}\left(\rho_{1} w_{1} \phi_{1}+\gamma_{1} \nabla w_{1} \cdot \nabla \phi_{1}\right) d x \\
& +\int_{\Omega_{2}}\left(\rho_{2} w_{2} \phi_{2}+\gamma_{2} \nabla w_{2} \cdot \nabla \phi_{2}\right) d x \\
\left(\left\{w_{1}, w_{2}\right\},\left\{\phi_{1}, \phi_{2}\right\}\right)_{H_{\mathrm{T}}^{2}}:=\int_{\Omega_{1}} \beta_{1} \Delta w_{1} \Delta \phi_{1} d x+\int_{\Omega_{2}} \beta_{2} \Delta w_{2} \Delta \phi_{2} d x & \\
(w, \phi)_{H_{\mathrm{D}}^{1}}:= & \int_{\Omega_{1}} \beta_{0} \nabla w \cdot \nabla \phi+\gamma_{0} w \phi d x+\int_{\Gamma_{1}} \beta_{0} \lambda w \phi d \Gamma \\
(w, \phi)_{H^{0}}:= & \int_{\Omega_{1}} \rho_{0} w \phi d x
\end{aligned}
$$

Let us denote the dual space of $H_{\mathrm{T}}^{s}$ by $H_{\mathrm{T}}^{-s}$ for $s=1,2$ and the dual space of $H_{\mathrm{D}}^{1}$ by $H_{\mathrm{D}}^{-1}$. The following Lemma shows that the norm given by the inner product in $H_{\mathrm{T}}^{2}$ is equivalent to the usual norm of $H^{2}\left(\Omega_{1}\right) \times H^{2}\left(\Omega_{2}\right)$.

Lemma 2.1. Let us take $(f, g)$ in $L^{2}\left(\Omega_{1}\right) \times L^{2}\left(\Omega_{2}\right)$. Then there exists a unique couple

$$
(u, v) \in H^{2}\left(\Omega_{1}\right) \times H^{2}\left(\Omega_{2}\right)
$$

solution of

$$
\Delta u=f \quad \text { on } \quad \Omega_{1}, \quad \Delta v=g \quad \text { on } \quad \Omega_{2},
$$

satisfying the boundary conditions

$$
u=0 \quad \text { on } \quad \Gamma_{1}, \quad v=0 \quad \text { on } \quad \Gamma_{2}, \quad u=v \quad \text { and } \quad \frac{\partial u}{\partial \nu}=\frac{\partial v}{\partial \nu} \quad \text { on } \quad \Gamma_{0} .
$$

Moreover, there exists $C>0$ such that

$$
\|u\|_{H^{2}\left(\Omega_{1}\right)}+\|v\|_{H^{2}\left(\Omega_{2}\right)} \leq C\left(\|f\|_{L^{2}\left(\Omega_{1}\right)}+\|g\|_{L^{2}\left(\Omega_{2}\right)}\right) .
$$

Proof. See [3].

The corresponding stationary transmission problem for the plate equation is given by the equations

$$
\Delta^{2} u=f \quad \text { in } \quad \Omega_{1}, \quad \Delta v^{2}=g \quad \text { in } \quad \Omega_{2},
$$

satisfying the boundary condition

$$
\begin{array}{r}
u=\frac{\partial u}{\partial \nu}=0 \quad \text { on } \quad \Gamma_{1}, \quad v=\frac{\partial v}{\partial \nu}=0 \quad \text { on } \quad \Gamma_{2}, \\
u=v, \quad \frac{\partial u}{\partial \nu}=\frac{\partial v}{\partial \nu}, \quad \beta_{1} \Delta u=\beta_{2} \Delta v, \quad \beta_{1} \frac{\partial \Delta u}{\partial \nu}=\beta_{2} \frac{\partial \Delta v}{\partial \nu} \quad \text { on } \quad \Gamma_{0} .
\end{array}
$$

To find the variational formulation associated to this problem we multiply the first equation of $(2.1)$ by $\beta_{1} \phi_{1}$ and the second by $\beta_{2} \phi_{2}$ with $\left(\phi_{1}, \phi_{2}\right) \in H_{\mathrm{T}}^{2}$; next we integrate by parts to obtain

$$
\int_{\Omega_{1}} \beta_{1} \Delta w_{1} \Delta \phi_{1} d x+\int_{\Omega_{2}} \beta_{2} \Delta w_{2} \Delta \phi_{2} d x=\int_{\Omega_{1}} \beta_{1} f \phi_{1} d x+\int_{\Omega_{2}} \beta_{2} g \phi_{2} d x .
$$

The existence and uniqueness of weak and strong solution for this problem is given by LEMMA 2.2. The following items hold: 
(1) If $(f, g) \in H_{\mathrm{T}}^{-2}$, then there exists a unique solution $(u, v) \in H_{\mathrm{T}}^{2}$ of $(2.1)-(2.3)$. Moreover, there exists $C>0$ such that

$$
\|(u, v)\|_{H_{\mathrm{T}}^{2}} \leq C\|(f, g)\|_{H_{\mathrm{T}}^{-2}} .
$$

(2) Let $m \in \mathbb{Z}_{0}^{+}$. If $(f, g) \in H^{m}\left(\Omega_{1}\right) \times H^{m}\left(\Omega_{2}\right)$, then there exists a unique solution $(u, v) \in H^{m+4}\left(\Omega_{1}\right) \times H^{m+4}\left(\Omega_{2}\right)$ of $(2.1)-(2.3)$. Moreover, there exists $C>0$ such that

$$
\|u\|_{H^{m+4}\left(\Omega_{1}\right)}+\|v\|_{H^{m+4}\left(\Omega_{2}\right)} \leq C\left(\|f\|_{H^{m}\left(\Omega_{1}\right)}+\|g\|_{H^{m}\left(\Omega_{2}\right)}\right) .
$$

(3) If $(f, g) \in H_{\mathrm{T}}^{-1}$, then there exists a unique solution $(u, v) \in H^{3}\left(\Omega_{1}\right) \times H^{3}\left(\Omega_{2}\right)$ of (2.1)-(2.3). Moreover, there exists $C>0$ such that

$$
\|(u, v)\|_{H^{3}\left(\Omega_{1}\right) \times H^{3}\left(\Omega_{2}\right)} \leq C\|(f, g)\|_{H_{\mathrm{T}}^{-1}} .
$$

Proof. Item 1 is a consequence of Lax-Milgram Theorem; item 2 , for the case $m=0$, can be found in [9] and item 3 is a consequence of the Interpolation Theory. Item 2 is a well known result of elliptic regularity (see [15] for the transmission problem for general elliptic equations). In Appendix A of this paper we give a simple proof of this item when $\beta_{1} / \beta_{2}$ is small or large.

Now, we shall write system (1.1)-(1.7) in the abstract form of semigroups following the ideas of Lagnese [4]. Let us consider the operators

$$
A_{0}: H_{\mathrm{T}}^{2} \rightarrow H_{\mathrm{T}}^{-2}, \quad A_{1}: H_{\mathrm{T}}^{1} \times H^{0} \rightarrow H_{\mathrm{T}}^{-1} \times H^{0} \quad \text { and } \quad B_{0}: H_{\mathrm{T}}^{2} \times H_{\mathrm{D}}^{1} \rightarrow H_{\mathrm{T}}^{-2} \times H_{\mathrm{D}}^{-1}
$$

given by

$$
\begin{aligned}
&\left\langle A_{0}\left\{w_{1}, w_{2}\right\},\left\{\phi_{1}, \phi_{2}\right\}\right\rangle:=\left(\left\{w_{1}, w_{2}\right\},\left\{\phi_{1}, \phi_{2}\right\}\right)_{H_{\mathrm{T}}^{2}}, \\
&\left\langle A_{1}\left\{w_{1}, w_{2}, w_{3}\right\},\left\{\phi_{1}, \phi_{2}, \phi_{3}\right\}\right\rangle:=\left(\left\{w_{1}, w_{2}\right\},\left\{\phi_{1}, \phi_{2}\right\}\right)_{H_{\mathrm{T}}^{1}}+\left(w_{3}, \phi_{3}\right)_{H^{0}}, \\
&\left\langle B_{0}\left\{w_{1}, w_{2}, w_{3}\right\},\left\{\phi_{1}, \phi_{2}, \phi_{3}\right\}\right\rangle:=\int_{\Omega_{1}} \mu\left(w_{3} \Delta \phi_{1}-\Delta w_{1} \phi_{3}\right) d x+\left(w_{3}, \phi_{3}\right)_{H_{\mathrm{D}}^{1}},
\end{aligned}
$$

and let us denote by

$$
B_{1}\left\{\phi_{1}, \phi_{2}\right\}:=\left\{A_{0}\left\{\phi_{1}, \phi_{2}\right\}, 0\right\}, \quad B_{2}\left\{\phi_{1}, \phi_{2}, \phi_{3}\right\}:=A_{0}\left\{\phi_{1}, \phi_{2}\right\} .
$$

Multiplying equations (1.1), (1.3), and (1.2) by $\phi_{1}, \phi_{2}$ and $\phi_{3}$ respectively with $\left\{\phi_{1}, \phi_{2}, \phi_{3}\right\} \in H_{\mathrm{T}}^{2} \times H_{\mathrm{D}}^{1}$ and performing an integration by parts yields

$$
\left\langle A_{1} \frac{d}{d t}\left\{u_{t}, v_{t}, \theta\right\},\left\{\phi_{1}, \phi_{2}, \phi_{3}\right\}\right\rangle=-\left\langle A_{0}\{u, v\},\left\{\phi_{1}, \phi_{2}\right\}\right\rangle-\left\langle B_{0}\left\{u_{t}, v_{t}, \theta\right\},\left\{\phi_{1}, \phi_{2}, \phi_{3}\right\}\right\rangle .
$$

The above identity can be written as

$$
A_{1} \frac{d}{d t}\left\{u_{t}, v_{t}, \theta\right\}=-B_{1}\{u, v\}-B_{0}\left\{u_{t}, v_{t}, \theta\right\} \quad \text { in } \quad H_{\mathrm{T}}^{-2} \times H_{\mathrm{D}}^{-1} .
$$

Taking into account that

$$
A_{0} \frac{d}{d t}\{u, v\}=A_{0}\left\{u_{t}, v_{t}\right\}
$$

and denoting by $w=\left(u, v, u_{t}, v_{t}, \theta\right)$, Eq. (2.4) can be written in the following matrix form

$$
\left(\begin{array}{cc}
A_{0} & 0 \\
0 & A_{1}
\end{array}\right) \frac{d w}{d t}+\left(\begin{array}{cc}
0 & -B_{2} \\
B_{1} & B_{0}
\end{array}\right) w=0 \text { in } H_{\mathrm{T}}^{-2} \times H_{\mathrm{T}}^{-2} \times H_{\mathrm{D}}^{-1} .
$$


Denoting by

$$
\mathbb{A}=\left(\begin{array}{cc}
A_{0} & 0 \\
0 & A_{1}
\end{array}\right), \quad \mathbb{B}=\left(\begin{array}{cc}
0 & -B_{2} \\
B_{1} & B_{0}
\end{array}\right)
$$

let us introduce the linear operator $\Lambda:=-\mathbb{A}^{-1} \mathbb{B}$ in $\mathcal{H}:=H_{\mathrm{T}}^{2} \times H_{\mathrm{T}}^{1} \times H^{0}$ with domain

$$
D(\Lambda)=\left\{w \in H_{\mathrm{T}}^{2} \times H_{\mathrm{T}}^{2} \times H_{\mathrm{D}}^{1}:\left\{A_{0}\left\{w_{1}, w_{2}\right\}, 0\right\}+B_{0}\left\{w_{3}, w_{4}, w_{5}\right\} \in H_{\mathrm{T}}^{-1} \times H^{0}\right\} .
$$

If we denote by

$$
\begin{gathered}
D_{0}:=\left\{w \in\left[H_{\mathrm{T}}^{2} \cap H^{3}\left(\Omega_{1}\right) \times H^{3}\left(\Omega_{2}\right)\right] \times H_{\mathrm{T}}^{2} \times\left[H^{2}\left(\Omega_{1}\right) \cap H_{\mathrm{D}}^{1}\right]:\right. \\
\left.\beta_{1} \Delta w_{1}=\beta_{2} \Delta w_{2} \text { on } \Gamma_{0} \text { and } \frac{\partial w_{5}}{\partial \nu}+\lambda w_{5}=0 \text { on } \Gamma_{1}\right\},
\end{gathered}
$$

it is clear that $D_{0} \subset D(\Lambda)$ and $D_{0}$ is dense in $\mathcal{H}$. The well-posedness of system (1.1)-(1.8) is given by the following Theorem.

TheOrem 2.3. The operator $\Lambda$ is the generator of a semigroup of class $C_{0}$ on $\mathcal{H}$.

Proof. We show that $\mathbb{A}^{-1} \mathbb{B}$ is maximal accretive; our conclusion will follow by the well-known Lumer-Phillips's Theorem.

$\mathbb{A}^{-1} \mathbb{B}$ is accretive: Let $w \in D(\Lambda)$, then

$$
\begin{aligned}
\left(\mathbb{A}^{-1} \mathbb{B} w, w\right)_{\mathcal{H}}= & \left(\mathbb{A}^{-1}\left[-A_{0}\left\{w_{3}, w_{4}\right\},\left\{A_{0}\left\{w_{1}, w_{2}\right\}, 0\right\}+B_{0}\left\{w_{3}, w_{4}, w_{5}\right\}\right], w\right)_{\mathcal{H}} \\
= & \left(\left\{w_{3}, w_{4}\right\}, A_{1}^{-1}\left[\left\{A_{0}\left\{w_{1}, w_{2}\right\}, 0\right\}+B_{0}\left\{w_{3}, w_{4}, w_{5}\right\}\right], w\right)_{\mathcal{H}} \\
= & -\left(\left\{w_{3}, w_{4}\right\},\left\{w_{1}, w_{2}\right\}\right)_{H_{T}^{2}}+\left\langle A_{0}\left\{w_{1}, w_{2}\right\},\left\{w_{3}, w_{4}\right\}\right\rangle_{H_{T}^{-2} \times H_{T}^{2}} \\
& +\left\langle B_{0}\left\{w_{3}, w_{4}, w_{5}\right\},\left\{w_{3}, w_{4}, w_{5}\right\}\right\rangle_{\left(H_{T}^{-2} \times H_{\mathrm{D}}^{-1}\right) \times\left(H_{T}^{2} \times H_{\mathrm{D}}^{1}\right)} \\
= & \left(w_{5}, w_{5}\right)_{H_{\mathrm{D}}^{1}} \geq 0 .
\end{aligned}
$$

$\mathbb{A}^{-1} \mathbb{B}$ is maximal: We need to show that the operator $I+\mathbb{A}^{-1} \mathbb{B}$ is surjective of $D(\Lambda)$ on $\mathcal{H}$. Since $\mathbb{A}$ is an isomorphism of $\mathcal{H}$ onto $\mathcal{H}^{\prime}$, it is sufficient to show that

$$
\mathbb{A}+\mathbb{B}: D(\Lambda) \rightarrow \mathcal{H}^{\prime} \quad \text { is surjective. }
$$

Given $f \in \mathcal{H}^{\prime}$, the equation $(\mathbb{A}+\mathbb{B}) w=f$ is equivalent to find $w \in D(\Lambda)$ such that

$$
\begin{aligned}
A_{0}\left\{w_{1}, w_{2}\right\}-A_{0}\left\{w_{3}, w_{4}\right\} & =\left(f_{1}, f_{2}\right) \in H_{\mathrm{T}}^{-2}, \\
A_{1}\left\{w_{3}, w_{4}, w_{5}\right\}+\left\{A_{0}\left\{w_{1}, w_{2}\right\}, 0\right\}+B_{0}\left\{w_{3}, w_{4}, w_{5}\right\} & =\left(f_{3}, f_{4}, f_{5}\right) \in H_{\mathrm{T}}^{-1} \times H^{0} .
\end{aligned}
$$

Substituting the first equation of (2.5) into the second equation, we get $A_{1}\left\{w_{3}, w_{4}, w_{5}\right\}+\left\{A_{0}\left\{w_{3}, w_{4}\right\}, 0\right\}+B_{0}\left\{w_{3}, w_{4}, w_{5}\right\}=\left(f_{3}-f_{1}, f_{4}-f_{2}, f_{5}\right) \in H_{\mathrm{T}}^{-2} \times H^{0}$.

Note that the operator $G: H_{\mathrm{T}}^{2} \times H_{\mathrm{D}}^{1} \rightarrow H_{\mathrm{T}}^{-2} \times H_{\mathrm{D}}^{-1}$, given by

$$
G\left\{w_{3}, w_{4}, w_{5}\right\}:=A_{1}\left\{w_{3}, w_{4}, w_{5}\right\}+\left\{A_{0}\left\{w_{3}, w_{4}\right\}, 0\right\}+B_{0}\left\{w_{3}, w_{4}, w_{5}\right\}
$$

is continuous and satisfies

$$
\begin{aligned}
\left\langle G\left\{w_{3}, w_{4}, w_{5}\right\},\left\{w_{3}, w_{4}, w_{5}\right\}\right\rangle_{\left(H_{\mathrm{T}}^{-2} \times H_{\mathrm{D}}^{-1}\right) \times\left(H_{\mathrm{T}}^{2} \times H_{\mathrm{D}}^{1}\right)}= & \left\|\left\{w_{3}, w_{4}, w_{5}\right\}\right\|_{H_{\mathrm{T}}^{1} \times H^{\circ}}^{2} \\
& +\left\|\left\{w_{3}, w_{4}\right\}\right\|_{H_{\mathrm{T}}^{2}}^{2}+\left\|w_{5}\right\|_{H_{\mathrm{D}}^{1}}^{2} \\
\geq & \left\|\left\{w_{3}, w_{4}, w_{5}\right\}\right\|_{H_{\mathrm{T}}^{2} \times H_{\mathrm{D}}^{1}}^{2}
\end{aligned}
$$


Therefore, the Lax-Milgram Theorem implies that there exists

$$
\left\{w_{3}, w_{4}, w_{5}\right\} \in H_{\mathrm{T}}^{2} \times H_{\mathrm{D}}^{1}
$$

such that relation (2.6) is satisfied. Since $A_{0}$ is a isomorphism of $H_{\mathrm{T}}^{2}$ on $H_{\mathrm{T}}^{-2}$, there exists

$$
\left\{w_{1}, w_{2}\right\} \in H_{\mathrm{T}}^{2}
$$

such that a first equation of (2.5) is satisfied. The second equation of (2.5) implies that

$$
\left\{A_{0}\left\{w_{1}, w_{2}\right\}, 0\right\}+B_{0}\left\{w_{3}, w_{4}, w_{5}\right\} \in H_{\mathrm{T}}^{-1} \times H^{0} ;
$$

therefore $w=\left(w_{1}, \ldots, w_{5}\right)$ given by $(2.7)-(2.8)$ belong in $D(\Lambda)$ and satisfy $(\mathbb{A}+\mathbb{B}) w=$ $f$.

3. Exponential decay. In this section we will show that the solution of the transmission problem (1.1)-(1.8) decays exponentially to zero as time goes to infinity. In Appendix B we prove that the functions in $D\left(\Lambda^{m}\right)$ are regular, so if we take regular initial data in $D\left(\Lambda^{m}\right)$, then the solution is regular, too. Therefore we can apply multiplier techniques to this system. Through this section we denote by $C$ a positive constant which will assume different values in different places. We shall assume that there exists $x_{0} \in \mathbb{R}^{n}$ such that the function $m(x): x-x_{0}$ satisfies

$$
\begin{array}{lll}
m \cdot \nu \geq \delta_{0} & \text { on } & \Gamma_{0}, \\
m \cdot \nu \leq 0 & \text { on } & \Gamma_{2},
\end{array}
$$

for $\delta_{0}>0$ small. Let us consider the following equation:

$$
\rho w_{t t}-\gamma \Delta w_{t t}+\beta \Delta^{2} w=0 \text { in } \Omega_{2} \times \mathbb{R}^{+},
$$

with boundary condition

$$
w=\frac{\partial w}{\partial \nu}=0 \quad \text { on } \quad\left(\Gamma_{2} \cup \Gamma_{0}\right) \times \mathbb{R}^{+} .
$$

The energy associated to this system is given by

$$
\hat{E}(t, w):=\frac{1}{2} \int_{\Omega_{2}} \rho\left|w_{t}\right|^{2}+\gamma\left|\nabla w_{t}\right|^{2}+\beta|\Delta w|^{2} d x .
$$

It is known that if $w$ is a weak solution of system (3.2)-(3.3), that is to say, $w \in$ $L^{\infty}\left(0, \infty ; H^{2}\left(\Omega_{2}\right)\right) \cap W^{1, \infty}\left(0, \infty ; H^{1}\left(\Omega_{2}\right)\right)$, then $\frac{d}{d t} \hat{E}(t, w)=0$ for any $t>0$; therefore the function

$$
t \mapsto \hat{E}(t, w) \quad \text { is constant. }
$$

Some properties for the solution of this system, such as regularity of the trace and observability inequality, are given by the following Lemma. A similar result also can be found in [2, Lemma 2.3].

LEmma 3.1. Let us suppose that $w \in L^{\infty}\left(0, \infty ; H^{2}\left(\Omega_{2}\right)\right) \cap W^{1, \infty}\left(0, \infty ; H^{1}\left(\Omega_{2}\right)\right)$ is a solution of (3.2)-(3.3); then the following properties hold: 
(1) There exists $C>0$ such that

$$
\int_{0}^{T} \int_{\Gamma_{2} \cup \Gamma_{0}}|\Delta w|^{2} d \Gamma d t \leq C \int_{0}^{T} \hat{E}(t, w) d t
$$

for $T$ large enough.

(2) There exists $C>0$ such that

$$
\int_{0}^{T} \hat{E}(t, w) d t \leq C \int_{0}^{T} \int_{\Gamma_{0}} m \cdot \nu|\Delta w|^{2} d \Gamma d t
$$

for $T$ large enough.

Proof. We will use multiplier techniques to show that the above inequalities hold for strong solutions, that is, for $w \in L^{\infty}\left(0, T ; H^{4}\left(\Omega_{2}\right)\right) \cap W^{1, \infty}\left(0, T ; H^{3}\left(\Omega_{2}\right)\right)$, so our conclusion will follow using standard density arguments. Let us consider the vector field $h=\left(h_{1}, \ldots, h_{n}\right) \in\left[C^{2}\left(\Omega_{2}\right)\right]^{n}$ such that $h(x)=\nu, \forall x \in \Gamma_{2} \cup \Gamma_{0}$. Multiplying Eq. (3.2) by $h \cdot \nabla w$ and integrating by parts, we get

$$
\begin{aligned}
& \frac{d}{d t} \int_{\Omega_{2}} \rho w_{t} h \cdot \nabla w+\gamma \nabla w_{t} \cdot \nabla(h \cdot \nabla w) d x \\
& =\frac{\beta}{2} \int_{\Gamma_{2} \cup \Gamma_{0}}|\Delta w|^{2} d \Gamma-\frac{1}{2} \int_{\Omega_{2}} \operatorname{div} h\left(\rho\left|w_{t}\right|^{2}+\gamma\left|\nabla w_{t}\right|^{2}-\beta|\Delta w|^{2}\right) d x \\
& \quad+\int_{\Omega_{2}}\left(\gamma \frac{\partial h_{k}}{\partial x_{j}} \frac{\partial w_{t}}{\partial x_{j}} \frac{\partial w_{t}}{\partial x_{k}}-2 \beta \Delta w \frac{\partial h_{k}}{\partial x_{j}} \frac{\partial w}{\partial x_{k}}\right) d x-\sum_{k=1}^{n} \int_{\Omega_{2}} \beta \Delta w \Delta h_{k} \frac{\partial w}{\partial x_{k}} d x
\end{aligned}
$$

Integrating over $[0, T]$ using Young's inequality and (3.4), we obtain that

$$
\begin{aligned}
\frac{\beta}{2} \int_{0}^{T} \int_{\Gamma_{2} \cup \Gamma_{0}}|\Delta w|^{2} d \Gamma d t & \leq C\left\{\int_{0}^{T} \hat{E}(t, w) d t+\hat{E}(T, w)+\hat{E}(0, w)\right\} \\
& \leq C\left\{\int_{0}^{T} \hat{E}(t, w) d t+\frac{2}{T} \int_{0}^{T} \hat{E}(t, w) d t\right\} .
\end{aligned}
$$

From this inequality the first part of this Lemma follows. To show the second part, we multiply Eq. (3.2) by $K w:=m(x) \cdot \nabla w+(n-3) w / 2$ and integrate by parts to obtain

$$
\begin{aligned}
\frac{d}{d t} \int_{\Omega}\left(\rho w_{t} K w+\gamma \nabla w_{t} \cdot \nabla K w\right) d x= & -\frac{1}{2} \int_{\Omega} 3 \rho\left|w_{t}\right|^{2}+\gamma\left|\nabla w_{t}\right|^{2}+\beta|\Delta w|^{2} d x \\
& +\frac{\beta}{2} \int_{\Gamma_{0}} m \cdot \nu|\Delta w|^{2} d \Gamma+\frac{\beta}{2} \int_{\Gamma_{2}} m \cdot \nu|\Delta w|^{2} d \Gamma .
\end{aligned}
$$

Integrating over $[0, T]$ using Young's inequality and hypothesis (3.1), we get

$$
\begin{aligned}
\int_{0}^{T} \hat{E}(t, w) d t & \leq \frac{\beta}{2} \int_{0}^{T} \int_{\Gamma_{0}} m \cdot \nu|\Delta w|^{2} d \Gamma d t+C\{\hat{E}(T, w)+\hat{E}(0, w)\} \\
& \leq \frac{\beta}{2} \int_{0}^{T} \int_{\Gamma_{0}} m \cdot \nu|\Delta w|^{2} d \Gamma d t+\frac{2 C}{T} \int_{0}^{T} \hat{E}(t, w) d t .
\end{aligned}
$$

Regrouping, we arrive at the following inequality:

$$
\left(1-\frac{2 C}{T}\right) \int_{0}^{T} \hat{E}(t, w) d t \leq \frac{\beta}{2} \int_{0}^{T} \int_{\Gamma_{0}} m \cdot \nu|\Delta w|^{2} d \Gamma d t .
$$


Now, taking $T$ large, our conclusion follows.

Let us consider the functionals $E$ (first order energy) and $F$ given by

$$
\begin{aligned}
E(t, u, v, \theta):= & \frac{1}{2} \int_{\Omega_{1}} \rho_{1}\left|u_{t}\right|^{2}+\gamma_{1}\left|\nabla u_{t}\right|^{2}+\beta_{1}|\Delta u|^{2}+\rho_{0}|\theta|^{2} d x \\
& +\frac{1}{2} \int_{\Omega_{2}} \rho_{2}\left|v_{t}\right|^{2}+\gamma_{2}\left|\nabla v_{t}\right|^{2}+\beta_{2}|\Delta v|^{2} d x, \\
F(t, u):= & \int_{\Omega_{1}}|\nabla u|^{2}+\left|u_{t}\right|^{2} d x,
\end{aligned}
$$

and denote by $\mathcal{E}$ and $\mathcal{F}$ as follows:

$$
\mathcal{E}(t, u, v, \theta):=\sum_{i=0}^{3} E\left(t, u^{(i)}, v^{(i)}, \theta^{(i)}\right), \quad \mathcal{F}(t, u):=\sum_{i=0}^{3} F\left(t, u^{(i)}\right),
$$

where $w^{(i)}=\frac{d^{i} w}{d t^{2}}, w^{(0)}=w$. The next Lemma will play an important role in the sequel. LEMMA 3.2. Given $\eta>0$, there exists a positive constant $C_{\eta}>0$ such that

$$
\int_{0}^{T} \mathcal{F}(t, u) d t \leq \eta \int_{0}^{T} \mathcal{E}(t, u, v, \theta) d t+C_{\eta} \sum_{i=0}^{3} \int_{0}^{T} \int_{\Omega_{1}} \beta_{0}\left|\nabla \theta^{(i)}\right|^{2} d x d t
$$

for any solution $(u, v, \theta)$ of $(1.1)-(1.8)$ such that $\left(u_{0}, v_{0}, u_{1}, v_{1}, \theta_{0}\right) \in D\left(\Lambda^{5}\right)$.

Proof. To establish our result we assume that the hypothesis of this Lemma is not true and derive a contradiction. Let us suppose that the inequality (3.6) does not hold, that is to say, there exist $\eta_{0}>0$ and $\left(u_{0}^{n}, v_{0}^{n}, u_{1}^{n}, v_{1}^{n}, \theta_{0}^{n}\right)_{n \in \mathbb{N}}$ in $D\left(\Lambda^{5}\right)$ such that the solutions $\left(u^{n}, v^{n}, \theta^{n}\right)$ of (1.1)-(1.8) satisfy

$$
\eta_{0} \int_{0}^{T} \mathcal{E}\left(t, u^{n}, v^{n}, \theta^{n}\right) d t+n \sum_{i=0}^{3} \int_{0}^{T} \int_{\Omega_{1}} \beta_{0}\left|\nabla \theta^{n(i)}\right|^{2} d x d t<\int_{0}^{T} \mathcal{F}\left(t, u^{n}\right) d t
$$

for any $n \in \mathbb{N}$. Without loss of generality, since the system (1.1)-(1.7) is linear, we can suppose that

$$
\int_{0}^{T} \mathcal{F}\left(t, u^{n}\right) d t=1
$$

Hence the inequality (3.7) can be written as

$$
\eta_{0} \int_{0}^{T} \mathcal{E}\left(t, u^{n}, v^{n}, \theta^{n}\right) d t+n \sum_{i=0}^{3} \int_{0}^{T} \int_{\Omega_{1}} \beta_{0}\left|\nabla \theta^{n(i)}\right|^{2} d x d t<1
$$

for any $n \in \mathbb{N}$. This inequality implies that $\left(u^{n(i)}, v^{n(i)}, \theta^{n(i)}\right)$ is bounded in $L^{2}\left(0, T ; H_{\mathrm{T}}^{2} \times\right.$ $H^{0}$ ) for $i=0, \ldots, 3$; therefore there exists a subsequence, which we still denote in the same way, such that

$$
\left(u^{n(i)}, v^{n(i)}, \theta^{n(i)}\right) \rightarrow\left(u^{(i)}, v^{(i)}, \theta^{(i)}\right) \quad \text { in } \quad L^{2}\left(0, T ; H_{\mathrm{T}}^{2} \times H^{0}\right) .
$$


Since $\left(u^{n(i)}, v^{n(i)}, \theta^{n(i)}\right)$ is a solution of $(1.1)-(1.7)$, then $\left(u^{(i)}, v^{(i)}, \theta^{(i)}\right)$ is a solution too, so it satisfies the equations

$$
\begin{aligned}
\rho_{1} u_{t t}-\gamma_{1} \Delta u_{t t}+\beta_{1} \Delta^{2} u+\mu \Delta \theta & =0 \text { in } \Omega_{1} \times \mathbb{R}^{+} \\
\rho_{0} \theta_{t}-\beta_{0} \Delta \theta+\gamma_{0} \theta-\mu \Delta u_{t} & =0 \text { in } \Omega_{1} \times \mathbb{R}^{+} \\
\rho_{2} v_{t t}-\gamma_{2} \Delta v_{t t}+\beta_{2} \Delta^{2} v & =0 \text { in } \Omega_{2} \times \mathbb{R}^{+}
\end{aligned}
$$

and the boundary conditions

$$
\begin{aligned}
& u=\frac{\partial u}{\partial \nu}=0 \quad \text { on } \quad \Gamma_{1} \times \mathbb{R}^{+}, \quad v=\frac{\partial v}{\partial \nu}=0 \quad \text { on } \quad \Gamma_{2} \times \mathbb{R}^{+}, \\
& u=v, \quad \frac{\partial u}{\partial \nu}=\frac{\partial v}{\partial \nu}, \quad \beta_{1} \Delta u=\beta_{2} \Delta v \quad \text { on } \quad \Gamma_{0} \times \mathbb{R}^{+}, \\
& -\gamma_{1} \frac{\partial u_{t t}}{\partial \nu}+\beta_{1} \frac{\partial \Delta u}{\partial \nu}+\mu \frac{\partial \theta}{\partial \nu}=-\gamma_{2} \frac{\partial v_{t t}}{\partial \nu}+\beta_{2} \frac{\partial \Delta v}{\partial \nu} \quad \text { on } \quad \Gamma_{0} \times \mathbb{R}^{+}, \\
& \theta=0 \quad \text { on } \quad \Gamma_{0} \times \mathbb{R}^{+}, \quad \frac{\partial \theta}{\partial \nu}+\lambda \theta=0 \quad \text { on } \quad \Gamma_{1} \times \mathbb{R}^{+} .
\end{aligned}
$$

Using (3.8) and Lions-Aubin's Compactness Theorem (see [7]), we can prove that

$$
\int_{0}^{T} \mathcal{F}(t, u) d t=1 .
$$

The inequality (3.9) implies that

$$
\nabla \theta=0 \quad \text { in } \quad \Omega_{1} \times \mathbb{R}^{+} .
$$

From Poincaré's inequality we have that $\theta=0$ in $\Omega_{1} \times \mathbb{R}^{+}$. Equation (3.11) implies that $\Delta u_{t}=0$ in $\Omega_{1} \times \mathbb{R}^{+}$. Differentiating Eq. (3.10) with respect to $t$ we obtain that $u_{t t t}=0$ in $\Omega_{1} \times \mathbb{R}^{+}$. Differentiating Eq. (3.12)--(3.14) in $t$ we have that $v_{t t t}$ is a weak solution of (3.2)-(3.3), satisfying

$$
\Delta v_{t t t}=\frac{\beta_{1}}{\beta_{2}} \Delta u_{t t t}=0 \quad \text { on } \quad \Gamma_{0} .
$$

Lemma 3.1 implies that $v_{t t t}=0$ in $\Omega_{2} \times \mathbb{R}^{+}$. Differentiating Eqs. (3.10)-(3.16) in $t$, we find that $\left(u_{t}, v_{t}\right)$ satisfies the hypothesis of Lemma 2.2 with $(f, g)=(0,0)$; therefore $\left(u_{t}, v_{t}\right)=(0,0)$. In these conditions, from equations (3.10)-(3.16) we conclude that $(u, v)=(0,0)$. This is contradictory to (3.17), hence our conclusion follows.

The exponential decay for regular solutions is given by the following theorem.

THEorem 3.3. Let us take $\left(u_{0}, v_{0}, u_{1}, v_{1}, \theta_{0}\right) \in D\left(\Lambda^{3}\right)$. If

$$
\rho_{1} \geq \rho_{2}, \quad \gamma_{1} \geq \gamma_{2} \quad \text { and } \quad \beta_{1} \leq \beta_{2},
$$

then there exist positive constants $C$ and $\kappa$ such that

$$
\mathcal{E}(t, u, v, \theta) \leq C \mathcal{E}(0, u, v, \theta) e^{-\kappa t},
$$

for any solution $(u, v, \theta)$ of $(1.1)-(1.8)$.

We shall prove this result for initial data $\left(u_{0}, v_{0}, u_{1}, v_{1}, \theta_{0}\right) \in D\left(\Lambda^{5}\right)$; the general case follows using a standard density argument. Let us denote by $B_{\delta}\left(\Omega_{2}\right):=\left\{x \in \mathbb{R}^{n}\right.$ : 
$\left.\inf _{y \in \Omega_{2}}|y-x|<\delta\right\}$ with $\delta>0$ small and let us consider the non-negative functions $\varphi_{i}, \psi_{i}, i=1, \ldots, 4$, of class $C^{\infty}\left(\mathbb{R}^{n}\right)$ given by

$$
\varphi_{i}(x)=\left\{\begin{array}{lll}
0 & \text { if } & x \in B_{i \delta / 2}\left(\Omega_{2}\right) \\
1 & \text { if } & x \in \mathbb{R}^{n} \backslash B_{i \delta}\left(\Omega_{2}\right)
\end{array} \quad, \quad \psi_{i}(x)=\left\{\begin{array}{lll}
1 & \text { if } & x \in B_{i \delta}\left(\Omega_{2}\right) \\
0 & \text { if } & x \in \mathbb{R}^{n} \backslash B_{2 i \delta}\left(\Omega_{2}\right) .
\end{array}\right.\right.
$$
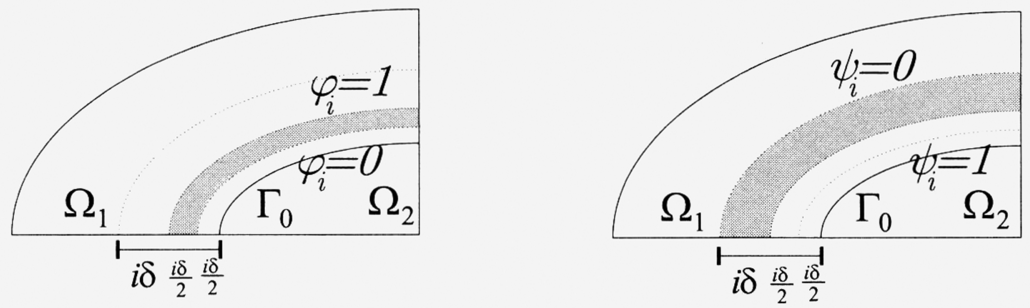

FIG. 2. The functions $\varphi_{i}, \psi_{i}$

Decomposing the set $\Omega_{1}$ by $\Omega_{1}=U_{i} \cup V_{i}$ with

$$
U_{i}=\Omega_{1} \backslash B_{i \delta}\left(\Omega_{2}\right), \quad V_{i}=\Omega_{1} \cap B_{i \delta}\left(\Omega_{2}\right) \text { for } i=1, \ldots, 4,
$$

we get that $\varphi_{i}=1$ in $U_{i}$ and $\psi_{i}=1$ in $V_{i}$. The proof of this Theorem will be a consequence of the following lemmas. Let us denote by $R_{1}$ the functional

$$
R_{1}(t, u, \theta):=\int_{\Omega_{1}} \rho_{0} \varphi_{1} \theta u_{t} d x .
$$

Lemma 3.4. Given $\eta>0$ there exists a positive constant $C_{\eta}$ such that

$$
\begin{aligned}
\frac{d}{d t} R_{1}(t, u, \theta) \leq & \eta\left\{\int_{\Gamma_{1}}|\Delta u|^{2} d \Gamma+\int_{\Omega_{1}}|\Delta u|^{2}+\left|\nabla u_{t}\right|^{2} d x\right\} \\
& +C_{\eta} \int_{\Omega_{1}}\left|u_{t}\right|^{2}+|\nabla \theta|^{2} d x-\mu \int_{U_{1}}\left|\nabla u_{t}\right|^{2} d x
\end{aligned}
$$

Proof. Multiplying Eq. (1.2) by $\varphi_{1} u_{t}$ and integrating by parts, we get

$$
\begin{aligned}
\frac{d}{d t} R_{1}(t, u, \theta)= & \rho_{0} \int_{\Omega_{1}} \varphi_{1} \theta u_{t t} d x-\int_{\Omega_{1}} \beta_{0} \nabla \theta \cdot \nabla\left(\varphi_{1} u_{t}\right)+\gamma_{0} \theta\left(\varphi_{1} u_{t}\right) d x \\
& -\mu \int_{\Omega_{1}} \varphi_{1}\left|\nabla u_{t}\right|^{2} d x-\mu \int_{\Omega_{1}} u_{t} \nabla \varphi_{1} \cdot \nabla u_{t} d x .
\end{aligned}
$$

Let $w$ be the solution of the stationary problem

$$
\begin{gathered}
\left(\rho_{1} I-\gamma_{1} \Delta\right) w=\rho_{0} \varphi_{1} \theta \text { in } \Omega_{1}, \\
w=0 \quad \text { on } \Gamma_{1}, \quad \frac{\partial w}{\partial \nu}=0 \text { on } \Gamma_{0} .
\end{gathered}
$$

From the Trace Theorem and the elliptic regularity it follows that there exists $C>0$ such that

$$
\|w\|_{H^{2}\left(\Omega_{1}\right)}^{2}+\int_{\Gamma_{1}}\left|\frac{\partial w}{\partial \nu}\right|^{2} d \Gamma \leq C \int_{\Omega_{1}}|\theta|^{2} d x
$$


Multiplying Eq. (1.1) by $w$ and performing an integration by parts, we get

$$
\rho_{0} \int_{\Omega_{1}} \varphi_{1} \theta u_{t t} d x=\beta_{1} \int_{\Gamma_{1}} \Delta u \frac{\partial w}{\partial \nu} d \Gamma-\beta_{1} \int_{\Omega_{1}} \Delta u \Delta w d x+\mu \int_{\Omega_{1}} \nabla \theta \cdot \nabla w d x
$$

substitution of this identity into (3.19) yields

$$
\begin{aligned}
\frac{d}{d t} R_{1}(t, u, \theta)= & \beta_{1} \int_{\Gamma_{1}} \Delta u \frac{\partial w}{\partial \nu} d \Gamma-\beta_{1} \int_{\Omega_{1}} \Delta u \Delta w d x+\mu \int_{\Omega_{1}} \nabla \theta \cdot \nabla w d x \\
& -\int_{\Omega_{1}} \beta_{0} \nabla \theta \cdot \nabla\left(\varphi_{1} u_{t}\right)+\gamma_{0} \theta\left(\varphi_{1} u_{t}\right) d x-\mu \int_{\Omega_{1}} \varphi_{1}\left|\nabla u_{t}\right|^{2} d x \\
& -\mu \int_{\Omega_{1}} u_{t} \nabla \varphi_{1} \cdot \nabla u_{t} d x
\end{aligned}
$$

Applying Young and Poincaré's inequalities and (3.20), our conclusion follows.

Let us consider a vector field $h=\left(h_{1}, \ldots, h_{n}\right) \in\left[C^{2}(\Omega)\right]^{n}$ satisfying

$$
h(x):=\left\{\begin{array}{lll}
-\nu(x) & \text { if } & x \in \Gamma_{1} \\
0 & \text { if } & x \in B_{\delta}\left(\Omega_{2}\right)
\end{array},\right.
$$

and let us introduce the following functionals:

$$
\begin{aligned}
J_{1}(t, u) & :=\int_{\Omega_{1}} \rho_{1} u_{t} h \cdot \nabla u+\gamma_{1} \nabla u_{t} \cdot \nabla(h \cdot \nabla u) d x, \\
R_{2}(t, u, \theta) & :=R_{1}(t, u, \theta)+\frac{2 \eta}{\beta_{1}} J_{1}(t, u) .
\end{aligned}
$$

LEMma 3.5. Given $\eta>0$ there exists a positive constant $C_{\eta}>0$ such that

$$
\begin{aligned}
\frac{d}{d t} R_{2}(t, u, \theta) & \leq \eta C\left\{\int_{\Omega_{1}}|\Delta u|^{2}+\left|\nabla u_{t}\right|^{2} d x\right\}+\int_{\Omega_{2}}|\Delta v|^{2} d x \\
& +C_{\eta} \int_{\Omega_{1}}\left|u_{t}\right|^{2}+|\nabla \theta|^{2} d x-\mu \int_{U_{1}}\left|\nabla u_{t}\right|^{2} d x .
\end{aligned}
$$

Proof. Multiplying Eq. (1.1) by $h \cdot \nabla u$ and integrating by parts, we get

$$
\begin{aligned}
\frac{d}{d t} J_{1}(t, u)= & -\frac{\rho_{1}}{2} \int_{\Omega_{1}} \operatorname{div} h\left|u_{t}\right|^{2} d x+\gamma_{1} \int_{\Omega_{1}} \nabla u_{t} \cdot \nabla h_{i} \frac{\partial u_{t}}{\partial x_{i}} d x \\
& -\frac{\gamma_{1}}{2} \int_{\Omega_{1}} \operatorname{div} h\left|\nabla u_{t}\right|^{2} d x-\frac{\beta_{1}}{2} \int_{\Gamma_{1}}|\Delta u|^{2} d \Gamma+\frac{\beta_{1}}{2} \int_{\Omega_{1}} \operatorname{div} h|\Delta u|^{2} d x \\
& -\beta_{1} \int_{\Omega_{1}} \Delta u\left(2 \nabla h_{i} \cdot \nabla \frac{\partial u}{\partial x_{i}}+\Delta h_{i} \frac{\partial u}{\partial x_{i}}\right) d x+\mu \int_{\Omega_{1}} \nabla \theta \cdot \nabla(h \cdot \nabla u) d x .
\end{aligned}
$$

Using Young and Poincaré's inequality once more, we find that

$$
\begin{aligned}
\frac{d}{d t} J_{1}(t, u) \leq & -\frac{\beta_{1}}{2} \int_{\Gamma_{1}}|\Delta u|^{2} d \Gamma \\
& +C\left\{\int_{\Omega_{1}}\left(|\Delta u|^{2}+\left|\nabla u_{t}\right|^{2}+|\nabla \theta|^{2}\right) d x+\int_{\Omega_{2}}|\Delta v|^{2} d x\right\} .
\end{aligned}
$$


Combining this inequality with Lemma 3.4, we conclude that

$$
\begin{aligned}
\frac{d}{d t} R_{2}(t, u, \theta) & \leq \eta C\left\{\int_{\Omega_{1}}|\Delta u|^{2}+\left|\nabla u_{t}\right|^{2} d x\right\}+\int_{\Omega_{2}}|\Delta v|^{2} d x \\
& +C_{\eta} \int_{\Omega_{1}}\left|u_{t}\right|^{2}+|\nabla \theta|^{2} d x-\mu \int_{U_{1}}\left|\nabla u_{t}\right|^{2} d x .
\end{aligned}
$$

This completes the proof.

Let us introduce the following functionals:

$$
\begin{aligned}
J_{2}(t, u) & :=\int_{\Omega} \rho_{1} u_{t} \varphi_{2} u+\gamma_{1} \nabla u_{t} \cdot \nabla\left(\varphi_{2} u\right) d x, \\
R_{3}(t, u, \theta) & :=R_{2}(t, u, \theta)+k_{0} J_{2}(t, u),
\end{aligned}
$$

where the constant $k_{0}$ is given by $(3.22)$.

LEMma 3.6. Given $\eta>0$, there exists a positive constant $C_{\eta}>0$ such that

$$
\begin{aligned}
\frac{d}{d t} R_{3}(t, u, \theta) \leq & -\frac{k_{0} \beta_{1}}{2} \int_{U_{2}}|\Delta u|^{2}+\left|\nabla u_{t}\right|^{2} d x+C_{\eta} \int_{\Omega_{1}}|\nabla u|^{2}+\left|u_{t}\right|^{2}+|\nabla \theta|^{2} d x \\
& +\eta C\left\{\int_{\Omega_{1}}|\Delta u|^{2}+\left|\nabla u_{t}\right|^{2} d x\right\}+\int_{\Omega_{2}}|\Delta v|^{2} d x
\end{aligned}
$$

Proof. Multiplying Eq. (1.1) by $\varphi_{2} u$ and integrating by parts, we get

$$
\begin{aligned}
\frac{d}{d t} J_{2}(t, u)= & \rho_{1} \int_{\Omega_{1}} \varphi_{2}\left|u_{t}\right|^{2} d x+\gamma_{1} \int_{\Omega_{1}} \nabla \varphi_{2} \cdot \nabla u_{t} u_{t} d x+\gamma_{1} \int_{\Omega_{1}} \varphi_{2}\left|\nabla u_{t}\right|^{2} d x \\
& -\beta_{1} \int_{\Omega_{1}} \varphi_{2}|\Delta u|^{2} d x-\beta_{1} \int_{\Omega_{1}} \Delta u\left(2 \nabla \varphi_{2} \cdot \nabla u+\Delta \varphi_{2} u\right) d x \\
& -\mu \int_{\Omega_{1}} \theta \Delta\left(\varphi_{2} u\right) d x .
\end{aligned}
$$

It follows that

$$
\begin{aligned}
\frac{d}{d t} J_{2}(t, u) \leq & -\frac{\beta_{1}}{2} \int_{U_{2}}|\Delta u|^{2} d x+C \int_{U_{1}}\left|\nabla u_{t}\right|^{2} d x \\
& +\eta \int_{\Omega_{1}}|\Delta u|^{2} d x+C_{\eta} \int_{\Omega_{1}}|\nabla u|^{2}+|\nabla \theta|^{2} d x
\end{aligned}
$$

Let $k_{0}$ be a positive constant satisfying

$$
\mu=k_{0}\left(\frac{\beta_{1}}{2}+C\right) .
$$

From Lemma 3.5 and inequality (3.21), we find that

$$
\begin{aligned}
\frac{d}{d t} R_{3}(t, u, \theta) \leq & -\frac{k_{0} \beta_{1}}{2} \int_{U_{2}}|\Delta u|^{2}+\left|\nabla u_{t}\right|^{2} d x+C_{\eta} \int_{\Omega_{1}}|\nabla u|^{2}+\left|u_{t}\right|^{2}+|\nabla \theta|^{2} d x \\
& +\eta C\left\{\int_{\Omega_{1}}|\Delta u|^{2}+\left|\nabla u_{t}\right|^{2} d x\right\}+\int_{\Omega_{2}}|\Delta v|^{2} d x
\end{aligned}
$$

from where our conclusion follows. 
Let us denote by $K u:=m \cdot \nabla u+(n-3) u / 2$ and let us consider the following functionals:

$$
\begin{aligned}
J_{3}(t, u, v):= & \int_{\Omega_{1}} \rho_{1} u_{t} \psi_{4} K u+\gamma_{1} \nabla u_{t} \cdot \nabla\left(\psi_{4} K u\right) d x \\
& +\int_{\Omega_{2}} \rho_{2} v_{t} \psi_{4} K v+\gamma_{2} \nabla v_{t} \cdot \nabla\left(\psi_{4} K v\right) d x, \\
R_{4}(t, u, v, \theta):= & R_{3}(t, u, \theta)+k_{1} J_{3}(t, u, v),
\end{aligned}
$$

where the constant $k_{1}$ is given by (3.25).

LEMmA 3.7. There exist positive constants $k_{2}$ and $C$ such that

$$
\frac{d}{d t} R_{4}(t, u, v, \theta) \leq-\frac{k_{2}}{2} E(t, u, v, \theta)+C \int_{\Omega_{1}}\left\{|\nabla u|^{2}+\left|u_{t}\right|^{2}+|\nabla \theta|^{2}\right\} d x .
$$

Proof. Multiplying Eq. (1.1) by $\psi_{4}(x) K u$, Eq. (1.3) by $\psi_{4}(x) K v$, and integrating by parts, we get

$$
\begin{aligned}
\frac{d}{d t} & J_{3}(t, u, v) \\
& =-\frac{\left(\rho_{1}-\rho_{2}\right)}{2} \int_{\Gamma_{0}} m \cdot \nu\left|u_{t}\right|^{2} d \Gamma-\frac{\left(\gamma_{1}-\gamma_{2}\right)}{2} \int_{\Gamma_{0}} m \cdot \nu\left|\nabla u_{t}\right|^{2} d \Gamma \\
& -\beta_{1} \int_{\Gamma_{0}} \Delta u m \cdot \frac{\partial \nabla(u-v)}{\partial \nu} d \Gamma+\frac{\beta_{1}}{2} \int_{\Gamma_{0}} m \cdot \nu|\Delta u|^{2} d \Gamma-\frac{\beta_{2}}{2} \int_{\Gamma_{0}} m \cdot \nu|\Delta v|^{2} d \Gamma \\
& +\mu \int_{\Omega_{1}} \nabla \theta \cdot \nabla\left(\psi_{4} K u\right) d x-\frac{3 \rho_{1}}{2} \int_{V_{4}}\left|u_{t}\right|^{2} d x-\frac{\gamma_{1}}{2} \int_{V_{4}}\left|\nabla u_{t}\right|^{2} d x-\frac{\beta_{1}}{2} \int_{V_{4}}|\Delta u|^{2} d x \\
& -\frac{\rho_{1}}{2} \int_{U_{4}} \xi\left|u_{t}\right|^{2} d x+\gamma_{1} \int_{U_{4}} \nabla u_{t} \cdot \nabla\left(\psi_{4} m_{i}\right) \frac{\partial u_{t}}{\partial x_{i}} d x+\frac{(n-3) \gamma_{1}}{2} \int_{U_{4}} \nabla u_{t} \cdot \nabla \psi_{4} u_{t} d x \\
& -\frac{\gamma_{1}}{2} \int_{U_{4}} \xi\left|\nabla u_{t}\right|^{2} d x+\frac{\beta_{1}}{2} \int_{U_{4}} \xi|\Delta u|^{2} d x-\beta_{1} \int_{U_{4}} \frac{\partial u}{\partial x_{i}} \Delta u \Delta\left(\psi_{4} m_{i}\right) d x \\
& -2 \beta_{1} \int_{U_{4}} \Delta u \nabla \frac{\partial u}{\partial x_{i}} \cdot \nabla\left(\psi_{4} m_{i}\right) d x-\frac{(n-3) \beta_{1}}{2} \int_{U_{4}} \Delta u\left(\Delta \psi_{4} u+2 \nabla u \cdot \nabla \psi_{4}\right) d x \\
& +\frac{1}{2} \int_{\Gamma_{2}} m \cdot \nu\left(\rho_{2}\left|v_{t}\right|^{2}+\gamma_{2}\left|\nabla v_{t}\right|^{2}+\beta_{2}|\Delta v|^{2}\right) d \Gamma-\frac{3 \rho_{2}}{2} \int_{\Omega_{2}}\left|v_{t}\right|^{2} d x \\
& -\frac{\gamma_{2}}{2} \int_{\Omega_{2}}\left|\nabla v_{t}\right|^{2} d x-\frac{\beta_{2}}{2} \int_{\Omega_{2}}|\Delta v|^{2} d x,
\end{aligned}
$$

where $\xi:=\operatorname{div}\left(\psi_{4} m\right)-(n-3) \psi_{4}$. The transmission boundary conditions imply that

$$
\begin{aligned}
-\beta_{1} \int_{\Gamma_{0}} \Delta u m \cdot \frac{\partial \nabla(u-v)}{\partial \nu} d \Gamma & =-\beta_{1} \int_{\Gamma_{0}} \Delta u m_{i} \nu_{j} \frac{\partial^{2}(u-v)}{\partial x_{i} \partial x_{j}} d \Gamma \\
& =-\beta_{1} \int_{\Gamma_{0}} \Delta u m_{i} \nu_{i} \nu_{j}^{2} \Delta(u-v) d \Gamma \\
& =-\beta_{1} \int_{\Gamma_{0}} \Delta u m \cdot \nu(\Delta u-\Delta v) d \Gamma \\
& \leq-\frac{\beta_{1}}{2} \int_{\Gamma_{0}} m \cdot \nu\left(|\Delta u|^{2}-|\Delta v|^{2}\right) d \Gamma
\end{aligned}
$$


Substitution of the above inequality into the above identity (3.23), and using Young's inequality, relations (3.18), and Lemma 2.1, we arrive at

$$
\begin{aligned}
\frac{d}{d t} J_{3}(t, u, v) \leq & \eta\left\{\int_{\Omega_{1}}|\Delta u|^{2} d x+\int_{\Omega_{2}}|\Delta v|^{2} d x\right\}+C_{\eta} \int_{\Omega_{1}}|\nabla \theta|^{2} d x \\
& +C \int_{U_{2}}\left|u_{t}\right|^{2}+\left|\nabla u_{t}\right|^{2}+|\Delta u|^{2} d x+C \int_{\Omega_{1}}|\nabla u|^{2} d x \\
& -\frac{3 \rho_{1}}{2} \int_{V_{4}}\left|u_{t}\right|^{2} d x-\frac{\gamma_{1}}{2} \int_{V_{4}}\left|\nabla u_{t}\right|^{2} d x-\frac{\beta_{1}}{2} \int_{V_{4}}|\Delta u|^{2} d x \\
& -\frac{3 \rho_{2}}{2} \int_{\Omega_{2}}\left|v_{t}\right|^{2} d x-\frac{\gamma_{2}}{2} \int_{\Omega_{2}}\left|\nabla v_{t}\right|^{2} d x-\frac{\beta_{2}}{2} \int_{\Omega_{2}}|\Delta v|^{2} d x .
\end{aligned}
$$

Let $k_{1}$ be a positive constant such that

$$
\frac{k_{0} \beta_{1}}{2}=k_{1}(C+1)
$$

From Lemma 3.6 and inequality (3.24), it follows that the functional $R_{4}$ satisfies

$$
\begin{aligned}
\frac{d}{d t} R_{4}(t, u, v, \theta) \leq & \eta C\left\{\int_{\Omega_{1}}\left(|\Delta u|^{2}+\left|\nabla u_{t}\right|^{2}\right) d x+\int_{\Omega_{2}}|\Delta v|^{2} d x\right\} \\
& +C_{\eta} \int_{\Omega_{1}}\left\{|\nabla u|^{2}+\left|u_{t}\right|^{2}+|\nabla \theta|^{2}\right\} d x \\
& -k_{1} \int_{U_{2}}\left(\left|u_{t}\right|^{2}+\left|\nabla u_{t}\right|^{2}+|\Delta u|^{2}\right) d x \\
& -\frac{3 k_{1} \rho_{1}}{2} \int_{V_{4}}\left|u_{t}\right|^{2} d x-\frac{k_{1} \gamma_{1}}{2} \int_{V_{4}}\left|\nabla u_{t}\right|^{2} d x-\frac{k_{1} \beta_{1}}{2} \int_{V_{4}}|\Delta u|^{2} d x \\
& -\frac{3 k_{1} \rho_{2}}{2} \int_{\Omega_{2}}\left|v_{t}\right|^{2} d x-\frac{k_{1} \gamma_{2}}{2} \int_{\Omega_{2}}\left|\nabla v_{t}\right|^{2} d x-\frac{k_{1} \beta_{2}}{2} \int_{\Omega_{2}}|\Delta v|^{2} d x .
\end{aligned}
$$

Since $V_{4} \supset V_{2}$, we have that $\Omega_{1}=U_{2} \cap V_{4}$. Therefore, there exists a small positive constant $k_{2}$ such that

$$
\begin{aligned}
\frac{d}{d t} R_{4}(t, u, v, \theta) \leq & \eta C\left\{\int_{\Omega_{1}}\left(|\Delta u|^{2}+\left|\nabla u_{t}\right|^{2}\right) d x+\int_{\Omega_{2}}|\Delta v|^{2} d x\right\} \\
& +C_{\eta} \int_{\Omega_{1}}\left\{|\nabla u|^{2}+\left|u_{t}\right|^{2}+|\nabla \theta|^{2}\right\} d x-k_{2} E(t, u, v, \theta) .
\end{aligned}
$$

Taking $\eta$ small enough, our conclusion follows.

Proof of Theorem 3.3: Multiplying Eq. (1.1) by $u_{t}$, Eq. (1.2) by $\theta$, Eq. (1.3) by $v_{t}$, and performing an integration by parts, we obtain that

$$
\frac{d}{d t} E(t, u, v, \theta)=-\int_{\Omega_{1}} \beta_{0}|\nabla \theta|^{2}+\gamma_{0}|\theta|^{2} d x-\int_{\Gamma_{1}} \beta_{0} \lambda|\theta|^{2} d \Gamma .
$$

Let us define the Lyapunov's functional $L$, given by

$$
L(t, u, v, \theta):=N E(t, u, v, \theta)+R_{4}(t, u, v, \theta),
$$


where $N$ denotes a large positive constant to be fixed later. Taking $N$ large and combining Lemma 3.7 and identity (3.26), we get

$$
\frac{d}{d t} L(t, u, v, \theta) \leq-\frac{k_{2}}{2} E(t, u, v, \theta)-\frac{N}{2} \int_{\Omega_{1}} \beta_{0}|\nabla \theta|^{2} d x+C F(t, u) .
$$

Since the system $(1.1)-(1.8)$ is linear and $\left(u_{0}, v_{0}, u_{1}, v_{1}, \theta_{0}\right) \in D\left(\Lambda^{5}\right)$, then $\left(u^{(i)}, v^{(i)}, \theta^{(i)}\right)$ are strong solutions for $i=1,2,3$; therefore, using the same procedure, we have

$$
\frac{d}{d t} L\left(t, u^{(i)}, v^{(i)}, \theta^{(i)}\right) \leq-\frac{k_{2}}{2} E\left(t, u^{(i)}, v^{(i)}, \theta^{(i)}\right)-\frac{N}{2} \int_{\Omega_{1}} \beta_{0}\left|\nabla \theta^{(i)}\right|^{2} d x+C F\left(t, u^{(i)}\right) .
$$

Denoting by $\mathcal{L}(t, u, v, \theta)=\sum_{i=1}^{3} L\left(t, u^{(i)}, v^{(i)}, \theta^{(i)}\right)$, we conclude that

$$
\frac{d}{d t} \mathcal{L}(t, u, v, \theta) \leq-\frac{k_{2}}{2} \mathcal{E}(t, u, v, \theta)-\frac{N}{2} \sum_{i=0}^{3} \int_{\Omega_{1}} \beta_{0}\left|\nabla \theta^{(i)}\right|^{2} d x+C \mathcal{F}(t, u),
$$

where $\mathcal{E}$ and $\mathcal{F}$ are given by (3.5). Integrating over $[0, T]$, we get

$$
\begin{aligned}
\mathcal{L}(T, u, v, \theta) \leq & \mathcal{L}(0, u, v, \theta)-\frac{k_{2}}{2} \int_{0}^{T} \mathcal{E}(t, u, v, \theta) d t \\
& -\frac{N}{2} \sum_{i=0}^{3} \int_{0}^{T} \int_{\Omega_{1}} \beta_{0}\left|\nabla \theta^{(i)}\right|^{2} d x d t+C \int_{0}^{T} \mathcal{F}(t, u) d t .
\end{aligned}
$$

Using Lemma 3.2 with $\eta$ small enough and taking $N$ large enough, we obtain that

$$
\begin{aligned}
\mathcal{L}(T, u, v, \theta) & \leq \mathcal{L}(0, u, v, \theta)-\frac{k_{2}}{4} \int_{0}^{T} \mathcal{E}(t, u, v, \theta) d t \\
& \leq \mathcal{L}(0, u, v, \theta)-\frac{k_{2} T}{4} \mathcal{E}(T, u, v, \theta) .
\end{aligned}
$$

Using Young's inequality, we can prove that

$$
\frac{N}{2} \mathcal{E}(t, u, v, \theta) \leq \mathcal{L}(t, u, v, \theta) \leq 2 N \mathcal{E}(t, u, v, \theta),
$$

for $N$ large enough. Combining (3.27) and (3.28), we conclude that

$$
\mathcal{L}(T, u, v, \theta) \leq \mathcal{L}(0, u, v, \theta)-\frac{k_{2} T}{8 N} \mathcal{L}(T, u, v, \theta) .
$$

Denoting by $\alpha:=\left(1+k_{2} T /(8 N)\right)^{-1}$, this inequality can be written as

$$
\mathcal{L}(T, u, v, \theta) \leq \alpha \mathcal{L}(0, u, v, \theta) .
$$

Since $\alpha \in] 0,1$, the semigroup property of the system (1.1)-(1.7) implies that there exist positive constants $C$ and $\kappa$ such that

$$
\mathcal{L}(t, u, v, \theta) \leq C \mathcal{L}(0, u, v, \theta) e^{-\kappa t} .
$$

From (3.28) we conclude that

$$
\mathcal{E}(t, u, v, \theta) \leq C \mathcal{E}(0, u, v, \theta) e^{-\kappa t},
$$

from where our conclusions follow. 
COROLlary 3.8. If $\left(u_{0}, v_{0}, u_{1}, v_{1}, \theta_{0}\right) \in H_{\mathrm{T}}^{2} \times H_{\mathrm{T}}^{1} \times H^{0}$, then there exist positive constants $C, \kappa$ such that

$$
E(t, u, v, \theta) \leq C E(0, u, v, \theta) e^{-\kappa t}
$$

for any weak solution $(u, v, \theta)$ of $(1.1)-(1.8)$.

Proof. Let us consider $\Theta_{0}$ the solution of the elliptic problem

$$
\begin{gathered}
-\beta_{0} \Delta \Theta_{0}+\gamma_{0} \Theta_{0}=\rho_{0} \theta_{0}-\mu \Delta u_{0} \text { in } \Omega_{1}, \\
\Theta_{0}=0 \text { on } \Gamma_{0}, \quad \frac{\partial \Theta_{0}}{\partial \nu}+\lambda \Theta_{0}=0 \text { on } \Gamma_{1},
\end{gathered}
$$

and let $\left(U_{0}, V_{0}\right)$ be the solution of the variational transmission problem

$$
\int_{\Omega_{1}} \beta_{1} \Delta U_{0} \Delta \varphi d x+\int_{\Omega_{2}} \beta_{2} \Delta V_{0} \Delta \psi d x=\langle f, \varphi\rangle+\langle g, \psi\rangle
$$

for every $(\varphi, \psi) \in H_{\mathrm{T}}^{2}$, where $(f, g) \in H_{\mathrm{T}}^{-1}$ is given by

$$
\begin{aligned}
& \langle f, \varphi\rangle=\int_{\Omega_{1}} \rho_{1} u_{1} \varphi+\gamma_{1} \nabla u_{1} \cdot \nabla \varphi+\mu \nabla \Theta_{0} \cdot \nabla \varphi d x \\
& \langle g, \psi\rangle=\int_{\Omega_{2}} \rho_{1} v_{1} \psi+\gamma_{1} \nabla v_{1} \cdot \nabla \psi d x .
\end{aligned}
$$

Let us define the following functions:

$$
\begin{aligned}
U(x, t) & :=\int_{0}^{t} u(x, s) d s+U_{0}(x), \\
V(x, t) & :=\int_{0}^{t} v(x, s) d s+V_{0}(x), \\
\Theta(x, t) & :=\int_{0}^{t} \theta(x, s) d s+\Theta_{0}(x) .
\end{aligned}
$$

Since system $(1.1)-(1.7)$ is linear, we have that $(U, V, \Theta)$ also is solution of this system; moreover, it verifies

$$
\left(U_{t}, V_{t}, \Theta_{t}\right)=(u, v, \theta) \quad \text { and } \quad(U(0), V(0), \Theta(0))=\left(U_{0}, V_{0}, \Theta_{0}\right) .
$$

From Lemma 2.2 applied to (3.29)-(3.30), it follows that there exists a constant $C>0$ such that

$$
\sum_{i=0}^{1} E\left(0, U^{(i)}, V^{(i)}, \Theta^{(i)}\right) \leq C E(0, u, v, \theta)
$$

Using the same above reasoning, we can make a solution $(U, V, \Theta)$ such that

$$
\left(U^{(3)}, V^{(3)}, \Theta^{(3)}\right)=(u, v, \theta) \text { and } \sum_{i=0}^{3} E\left(0, U^{(i)}, V^{(i)}, \Theta^{(i)}\right) \leq C E(0, u, v, \theta) .
$$

Applying Theorem 3.3 to $(U, V, \Theta)$, we get that

$$
\sum_{i=0}^{3} E\left(t, U^{(i)}, V^{(i)}, \Theta^{(i)}\right) \leq C \sum_{i=0}^{3} E\left(0, U^{(i)}, V^{(i)}, \Theta^{(i)}\right) e^{-k t}
$$


In view of $(3.31)$, we conclude that

$$
E(t, u, v, \theta) \leq C E(0, u, v, \theta) e^{-k t} .
$$

This completes the proof.

Appendix A. In this section we give a simple proof of the elliptic regularity for the stationary transmission problem.

Lemma 3.9. Let $\lambda$ be given by (A.6). If $\beta_{1} / \beta_{2}$ satisfies

$$
\frac{\beta_{1}}{\beta_{2}}<\frac{1}{\lambda} \quad \text { or } \quad \frac{\beta_{1}}{\beta_{2}}>\lambda,
$$

then item 2 of Lemma 2.2 holds.

Proof. Let us take $(f, g) \in H^{m}\left(\Omega_{1}\right) \times H^{m}\left(\Omega_{2}\right)$ and $(w, z) \in \mathbb{I}^{m}:=H^{m+7 / 2}\left(\Gamma_{0}\right) \times$ $H^{m+5 / 2}\left(\Gamma_{0}\right)$. Consider $u \in H^{m+4}\left(\Omega_{1}\right)$ a unique solution of

$$
\begin{gathered}
\Delta^{2} u=f \quad \text { in } \Omega_{1} \\
u=\frac{\partial u}{\partial \nu}=0 \quad \text { on } \Gamma_{1}, \quad u=w \text { and } \frac{\partial u}{\partial \nu}=z \text { on } \Gamma_{0} .
\end{gathered}
$$

Then $\left(\left.\Delta u\right|_{\Gamma_{0}},\left.\frac{\partial \Delta u}{\partial \nu}\right|_{\Gamma_{0}}\right) \in H^{m+3 / 2}\left(\Gamma_{0}\right) \times H^{m+1 / 2}\left(\Gamma_{0}\right)$. Now, let us consider $v \in H^{m+4}\left(\Omega_{2}\right)$ the unique solution of

$$
\begin{gathered}
\Delta^{2} v=g \quad \text { in } \Omega_{2}, \\
v=\frac{\partial v}{\partial \nu}=0 \quad \text { on } \quad \Gamma_{2}, \quad \Delta v=\frac{\beta_{1}}{\beta_{2}} \Delta u \quad \text { and } \quad \frac{\partial \Delta v}{\partial \nu}=\frac{\beta_{1}}{\beta_{2}} \frac{\partial \Delta u}{\partial \nu} \quad \text { on } \Gamma_{0} .
\end{gathered}
$$

Then $\left(\left.v\right|_{\Gamma_{0}},\left.\frac{\partial v}{\partial \nu}\right|_{\Gamma_{0}}\right) \in \mathbb{I}^{m}$. In these conditions we can define the operator $T_{f g}: \mathbb{I}^{m} \rightarrow \mathbb{I}^{m}$ given by

$$
(w, z) \mapsto T_{f g}(w, z):=\left(\left.v\right|_{\Gamma_{0}},\left.\frac{\partial v}{\partial \nu}\right|_{\Gamma_{0}}\right) .
$$

Our conclusion will follow, showing that there exists a fixed point to $T_{f g}$. Let us take $\left(w_{1}, z_{1}\right)$ and $\left(w_{2}, z_{2}\right)$ in $\mathbb{I}^{m}$ and consider $\left(u_{i}, v_{i}\right) \in H^{m+4}\left(\Omega_{1}\right) \times H^{m+4}\left(\Omega_{2}\right), i=1,2$ solutions of

$$
\begin{gathered}
\Delta^{2} u_{i}=f \quad \text { in } \Omega_{1}, \\
u_{i}=\frac{\partial u_{i}}{\partial \nu}=0 \quad \text { on } \quad \Gamma_{1}, \quad u_{i}=w_{i} \text { and } \frac{\partial u_{i}}{\partial \nu}=z_{i} \text { on } \Gamma_{0}, \\
\Delta^{2} v_{i}=g \quad \text { in } \Omega_{2}, \\
v_{i}=\frac{\partial v_{i}}{\partial \nu}=0 \quad \text { on } \Gamma_{2}, \quad \Delta v_{i}=\frac{\beta_{1}}{\beta_{2}} \Delta u_{i} \text { and } \frac{\partial \Delta v_{i}}{\partial \nu}=\frac{\beta_{1}}{\beta_{2}} \frac{\partial \Delta u_{i}}{\partial \nu} \text { on } \Gamma_{0},
\end{gathered}
$$

and denote by

$$
\begin{aligned}
(w, z) & :=\left(w_{1}, z_{1}\right)-\left(w_{2}, z_{2}\right), \\
u & :=u_{1}-u_{2}, \\
v & :=v_{1}-v_{2} .
\end{aligned}
$$

It is not difficult to show that $(u, v)$ satisfies (A.2)-(A.5) with $(f, g)=(0,0)$. Therefore

$$
T_{f g}\left(w_{1}, z_{1}\right)-T_{f g}\left(w_{2}, z_{2}\right)=T_{00}(w, z) .
$$


Moreover, from the Trace Theorem and elliptic regularity it follows that there exist $\lambda_{1}, \ldots, \lambda_{4}$ such that

$$
\begin{aligned}
\left\|T_{00}(w, z)\right\|_{\mathbb{I}^{m}} & \leq \lambda_{1} \frac{\beta_{1}}{\beta_{2}}\|v\|_{H^{m+4}\left(\Omega_{2}\right)} \\
& \leq \lambda_{1} \lambda_{2} \frac{\beta_{1}}{\beta_{2}}\left\|\left(\left.\Delta u\right|_{\Gamma_{0}},\left.\frac{\partial \Delta u}{\partial \nu}\right|_{\Gamma_{0}}\right)\right\|_{H^{m+3 / 2}\left(\Gamma_{0}\right) \times H^{m+1 / 2}\left(\Gamma_{0}\right)} \\
& \leq \lambda_{1} \lambda_{2} \lambda_{3} \frac{\beta_{1}}{\beta_{2}}\|u\|_{H^{m+4}\left(\Omega_{1}\right)} \\
& \leq \lambda_{1} \lambda_{2} \lambda_{3} \lambda_{4} \frac{\beta_{1}}{\beta_{2}}\|(w, z)\|_{\mathbb{I}^{m}} .
\end{aligned}
$$

This implies that

$$
\left\|T_{f g}\left(w_{1}, z_{1}\right)-T_{f g}\left(w_{2}, z_{2}\right)\right\|_{H^{\mathrm{I}^{m}}} \leq \lambda \frac{\beta_{1}}{\beta_{2}}\left\|\left(w_{1}, z_{1}\right)-\left(w_{2}, z_{2}\right)\right\|_{\mathbb{I}^{m}},
$$

where $\lambda=\lambda_{1} \lambda_{2} \lambda_{3} \lambda_{4}$. If $\lambda \frac{\beta_{1}}{\beta_{2}}<1$, we have that $T_{f g}$ has a unique fixed point in $\mathbb{I}^{m}$. Using this reasoning and swapping $u$ and $v$ in (A.2)-(A.5), we obtain the same result when $\lambda \frac{\beta_{2}}{\beta_{1}}<1$. Therefore $T_{f g}$ has a unique fixed point when $\beta_{1} / \beta_{2}$ satisfies (A.1).

Appendix B. In this section we prove that the functions belonging to $D\left(\Lambda^{m}\right)$ are regular.

Lemma 3.10. We have that $D(\Lambda)=D_{0}$ and for any $m \in \mathbb{N}$ with $m \geq 2$ we get

$$
D\left(\Lambda^{m}\right) \subset H^{m+2}\left(\Omega_{1}\right) \times H^{m+2}\left(\Omega_{2}\right) \times H^{m+1}\left(\Omega_{1}\right) \times H^{m+1}\left(\Omega_{2}\right) \times H^{m+1}\left(\Omega_{1}\right) .
$$

Proof. Let us take $w \in D(\Lambda)$, then $w \in H_{\mathrm{T}}^{2} \times H_{\mathrm{T}}^{2} \times H_{\mathrm{D}}^{1}$ and

$$
\left\{A_{0}\left\{w_{1}, w_{2}\right\}, 0\right\}+B_{0}\left\{w_{3}, w_{4}, w_{5}\right\}=\left\{f_{1}, f_{2}, f_{3}\right\} \in H_{\mathrm{T}}^{-1} \times H^{0} .
$$

Multiplying this equation by $\{0,0, \phi\} \in H_{\mathrm{T}}^{1} \times H_{\mathrm{D}}^{1}$, we obtain that

$$
\left(w_{5}, \phi\right)_{H_{\mathrm{D}}^{1}}=\left(f_{3}, \phi\right)_{H^{0}}+\int_{\Omega_{1}} \mu \Delta w_{3} \phi d x .
$$

Since $f_{3}+\mu \Delta w_{3} \in L^{2}\left(\Omega_{1}\right)$, elliptic regularity implies that

$$
w_{5} \in H^{2}\left(\Omega_{1}\right) \text { and } \frac{\partial w_{5}}{\partial \nu}+\lambda w_{5}=0 \text { on } \Gamma_{1} .
$$

On the other hand, multiplying Eq. (B.1) by $\left\{\phi_{1}, \phi_{2}, 0\right\} \in H_{\mathrm{T}}^{2} \times H^{0}$, we get

$$
\begin{aligned}
\left(\left\{w_{1}, w_{2}\right\},\left\{\phi_{1}, \phi_{2}\right\}\right)_{H_{\mathrm{T}}^{2}}= & \left\langle\left\{f_{1}, f_{2}\right\},\left\{\phi_{1}, \phi_{2}\right\}\right\rangle_{H_{\mathrm{T}}^{-1} \times H_{\mathrm{T}}^{1}} \\
& +\underbrace{\int_{\Omega_{1}} \mu \nabla w_{5} \cdot \nabla \phi_{1} d x}_{\left\langle F,\left\{\phi_{1}, \phi_{2}\right\}\right\rangle} \forall\left\{\phi_{1} \phi_{2}\right\} \in H_{\mathrm{T}}^{2} .
\end{aligned}
$$

Since $\left\{f_{1}, f_{2}\right\}+F \in H_{\mathrm{T}}^{-1}$, Lemma 2.2 implies that

$$
\left\{w_{1}, w_{2}\right\} \in H^{3}\left(\Omega_{1}\right) \times H^{3}\left(\Omega_{2}\right) \quad \text { and } \quad \beta_{1} \Delta w_{1}=\beta_{2} \Delta w_{2} \text { on } \Gamma_{0} .
$$


From (B.2)-(B.3) we have that $w \in D_{0}$; therefore, the first part of this Lemma is proved. We shall prove the second part using induction. Let $m \in \mathbb{N}$ with $m \geq 2$ and suppose that

$$
D\left(\Lambda^{m}\right) \subset H^{m+2}\left(\Omega_{1}\right) \times H^{m+2}\left(\Omega_{2}\right) \times H^{m+1}\left(\Omega_{1}\right) \times H^{m+1}\left(\Omega_{2}\right) \times H^{m+1}\left(\Omega_{1}\right),
$$

and let us take $w \in D\left(\Lambda^{m+1}\right)$ such that $\Lambda w=f \in D\left(\Lambda^{m}\right)$. Applying the operator $-\mathbb{A}$, we get

$$
\left(-A_{0}\left\{w_{3}, w_{4}\right\},\left\{A_{0}\left\{w_{1}, w_{2}\right\}, 0\right\}+B_{0}\left\{w_{3}, w_{4}, w_{5}\right\}\right)=-\left(A_{0}\left\{f_{1}, f_{2}\right\}, A_{1}\left\{f_{3}, f_{4}, f_{5}\right\}\right) .
$$

Hence we find that

$$
\left\{w_{3}, w_{4}\right\}=\left\{f_{1}, f_{2}\right\} \in H^{m+2}\left(\Omega_{1}\right) \times H^{m+2}\left(\Omega_{2}\right),
$$

and

$$
\left\{A_{0}\left\{w_{1}, w_{2}\right\}, 0\right\}+B_{0}\left\{w_{3}, w_{4}, w_{5}\right\}=-A_{1}\left\{f_{3}, f_{4}, f_{5}\right\} .
$$

Multiplying Eq. (B.5) by $\{0,0, \phi\} \in H_{\mathrm{T}}^{1} \times H_{\mathrm{D}}^{1}$, we obtain that

$$
\left(w_{5}, \phi\right)_{H_{\mathrm{D}}^{1}}=-\left(f_{5}, \phi\right)_{H^{0}}+\int_{\Omega_{1}} \mu \Delta w_{3} \phi d x
$$

Since $-f_{5}+\mu \Delta w_{3} \in H^{m}\left(\Omega_{1}\right)$, elliptic regularity implies that

$$
w_{5} \in H^{m+2}\left(\Omega_{1}\right) .
$$

Multiplying Eq. (B.5) by $\left\{\phi_{1}, \phi_{2}, 0\right\} \in H_{\mathrm{T}}^{2} \times H^{0}$, we get

$$
\left(\left\{w_{1}, w_{2}\right\},\left\{\phi_{1}, \phi_{2}\right\}\right)_{H_{\mathrm{T}}^{2}}=-\left(\left\{f_{3}, f_{4}\right\},\left\{\phi_{1}, \phi_{2}\right\}\right)_{H_{\mathrm{T}}^{1}}-\int_{\Omega_{1}} \mu w_{5} \Delta \phi_{1} d x .
$$

Since $\left\{f_{3}, f_{4}, w_{5}\right\} \in H^{m+1}\left(\Omega_{1}\right) \times H^{m+1}\left(\Omega_{2}\right) \times H^{m+1}\left(\Omega_{1}\right)$, we have that

$$
h:=\gamma_{1} \frac{\partial f_{3}}{\partial \nu}-\gamma_{2} \frac{\partial f_{4}}{\partial \nu}-\mu \frac{\partial w_{5}}{\partial \nu} \in H^{m-\frac{1}{2}}\left(\Gamma_{0}\right) .
$$

Let $z$ be a function such that

$$
z \in H^{m+3}\left(\Omega_{1}\right) \quad z=\frac{\partial z}{\partial \nu}=\Delta z=0 \quad \text { on } \quad \Gamma_{1} \cap \Gamma_{0} . \quad \frac{\partial \Delta z}{\partial \nu}=\left\{\begin{array}{lll}
0 & \text { on } \Gamma_{1} \\
\frac{1}{\beta_{1}} h & \text { on } & \Gamma_{0}
\end{array} .\right.
$$

and let us denote by $\left\{\tilde{w}_{1}, \tilde{w}_{2}\right\}$ the solution of the stationary problem

$$
\begin{aligned}
& \Delta^{2} \tilde{w}_{1}=F \quad:=\frac{1}{\beta_{1}}\left[-\rho_{1} f_{3}+\gamma_{1} \Delta f_{3}-\mu \Delta w_{5}\right]-\Delta^{2} z \quad \text { in } \Omega_{1}, \\
& \Delta^{2} \tilde{w}_{2}=G:=\frac{1}{\beta_{2}}\left[-\rho_{2} f_{4}+\gamma_{2} \Delta f_{4}\right] \text { in } \Omega_{2} .
\end{aligned}
$$

with boundary conditions

$$
\begin{array}{r}
\tilde{w}_{1}=\frac{\partial \tilde{w}_{1}}{\partial \nu}=0 \quad \text { on } \quad \Gamma_{1}, \quad \tilde{w}_{2}=\frac{\partial \tilde{w}_{2}}{\partial \nu}=0 \quad \text { on } \quad \Gamma_{2}, \\
\tilde{w}_{1}=\tilde{w}_{2}, \quad \frac{\partial \tilde{w}_{1}}{\partial \nu}=\frac{\partial \tilde{w}_{2}}{\partial \nu}, \quad \beta_{1} \Delta \tilde{w}_{1}=\beta_{2} \Delta \tilde{w}_{2}, \quad \beta_{1} \frac{\partial \Delta \tilde{w}_{1}}{\partial \nu}=\beta_{2} \frac{\partial \Delta \tilde{w}_{2}}{\partial \nu} \quad \text { on } \quad \Gamma_{0} .
\end{array}
$$

Since $\{F, G\} \in H^{m-1}\left(\Omega_{1}\right) \times H^{m-1}\left(\Omega_{2}\right)$, Lemma 2.2 implies that

$$
\left\{\tilde{w}_{1}, \tilde{w}_{2}\right\} \in H^{m+3}\left(\Omega_{1}\right) \times H^{m+3}\left(\Omega_{2}\right) .
$$


The solution of variational equation (B.7) is unique; therefore $w_{1}=z+\tilde{w}_{1}, w_{2}=\tilde{w}_{2}$. From (B.8)-(B.9) we have

$$
\left\{w_{1}, w_{2}\right\} \in H^{m+3}\left(\Omega_{1}\right) \times H^{m+3}\left(\Omega_{2}\right) .
$$

Now, from (B.4), (B.6), and (B.10), we conclude that

$$
w \in H^{m+3}\left(\Omega_{1}\right) \times H^{m+3}\left(\Omega_{2}\right) \times H^{m+2}\left(\Omega_{1}\right) \times H^{m+2}\left(\Omega_{2}\right) \times H^{m+2}\left(\Omega_{1}\right),
$$

which completes the proof.

\section{REFERENCES}

[1] M. Aassila, Exact boundary controllability of the plate equation, Diff. Int. Eq., 13 (2000), 14131428

[2] G. Avalos and I. Lasiecka, Exponential stability of a thermoelastic system with free boundary conditions without mechanical dissipation, SIAM J. Math. Anal., 29 (1998), 155-182.

[3] O. A. Ladyzhenskaya and N. N. Ural'tseva, Linear and quasilinear elliptic equations, Academic Press, New York, 1968.

[4] J. E. Lagnese, Boundary stabilization of thin plates, SIAM Studies in Applied Mathematics, Philadelphia, 1989.

[5] J. Lagnese, Boundary controllability in problems of transmission for a class of second order hyperbolic systems, ESAIM Control Optim. Calc. Var., 2 (1997), 343-357.

[6] J.L. Lions, Contrôlabilité exacte perturbations et stabilisation de systèmes distribués, Collection RMA, Masson Paris 1988 (tome 1).

[7] J.L. Lions, Quelques méthodes de résolution des problèmes aux limites non linéaires, Collection Études Mathématiques, Dunod Paris 1969.

[8] W. Liu and G. Williams, The Exponential Stability of the Problem of Transmission of the Wave Equation, Bull. Austral. Math. Soc., Vol. 57 (1998), 305-327.

[9] W. Liu and G. Williams, Exact controllability for problems of transmission of the plate equation with lower-order terms, Quart. Appl. Math., f58 (2000), 37-68.

[10] J. E. Muñoz Rivera and Ma To Fu, Exponential stability for a transmission problem, To appear.

[11] J. E. Muñoz Rivera and H. Portillo Oquendo, The transmission problem of viscoelastic waves, Acta Applicandae Mathematicae, 60 (2000), 1-21.

[12] J. E. Muñoz Rivera and H. Portillo Oquendo, The transmission problem of thermoelastic beams, Journal of Thermal Stresses, 24 (2001), 1137-1158.

[13] J. E. Muñoz Rivera and H. Portillo Oquendo, The transmission problem of viscoelastic beams, To appear in Advances in Mathematical Science and Applications.

[14] K. Oh, A theorical and experimental study of modal interactions in metallic and laminated composite plates, Ph.D. thesis, Virginia Polytechnic Institute and State University, Blacksburg, VA, 1994.

[15] M. Schechter, A generalization of the problem of transmission, Ann. Scuola Norm. Sup. Pisa, Ser III, 14 (1960), 207-236. 